\title{
Two special cases of the Rational Shuffle Conjecture
}

\author{
Emily Leven]" \\ Mathematics Department, University of California, San Diego, USA
}

\begin{abstract}
The Classical Shuffle Conjecture of Haglund et al. (2005) has a symmetric function side and a combinatorial side. The combinatorial side $q, t$-enumerates parking functions in the $n \times n$ lattice. The symmetric function side may be simply expressed as $\nabla e_{n}$, where $\nabla$ is the Macdonald eigen-operator introduced by Bergeron and Garsia (1999) and $e_{n}$ is the elementary symmetric function. The combinatorial side has been extended to parking functions in the $m \times n$ lattice for coprime $m, n$ by Hikita (2012). Recently, Gorsky and Negut have been able to extend the Shuffle Conjecture by combining their work (2012a, 2012b, 2013) (related to work of Schiffmann and Vasserot (2011, 2013)) with Hikita's combinatorial results. We prove this new conjecture for the cases $m=2$ and $n=2$.

Résumé. La Conjecture "Shuffle" Classique de Haglund et al. (2005) présente un côté fonctions symétriques et un côté combinatoire. Le côté combinatoire $q$, $t$-énumère les fonctions parking dans le $n \times n$ treillis. Le côté fonction symétriques peut être simplement exprimé comme $\nabla e_{n}$, où $\nabla$ est le Macdonald eigen-opérateur introduit par Bergeron et Garsia (1999) et $e_{n}$ est la fonction symétrique élémentaire. Le côté combinatoire a été étendu aux fonctions parking dans le $m \times n$ treillis pour coprime $m, n$ par Hikita (2012). Récemment, Gorsky et Negut ont pu étendre la Conjecture Shuffle en reliant leur travail (2012a, 2012b, 2013) avec le travail de Schiffmann et Vasserot $(2011,2013)$ et avec des résultats combinatoires de Hikita. Nous prouvons cette nouvelle conjecture pour les cas $m=2$ et $n=2$.
\end{abstract}

Keywords: parking functions, Shuffle Conjecture, Hall-Littlewood operators

\section{Introduction}

Let $m$ and $n$ be coprime. Using the definitions of Hikita (2012), parking functions and their statistics can be extended to the $m \times n$ lattice as follows. Here classical parking functions correspond to the case $m=n+1$. For an overview of classical parking functions and their statistics, see, for example, Garsia and Haiman (1996) and Haglund et al. (2005). We will refer to the objects defined here as rational in contrast to the classical case.

An $(m, n)$-Dyck path is a path in the $m \times n$ lattice which proceeds by north and east steps from $(0,0)$ to $(m, n)$ and which always remains weakly above the main diagonal $y=\frac{n}{m} x$. Note that the main diagonal cannot pass through any lattice points (besides $(0,0)$ and $(m, n)$ ) due to the co-primality of $m$ and $n$. In fact, the main diagonal cuts through $m+n-1$ cells of the $m \times n$ lattice. For example, see Figure 1 .

\footnotetext{
*Email: esergel@ucsd.edu. Supported by NSF grant DGE-1144086 


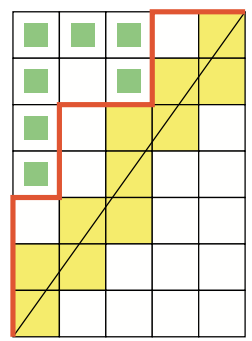

Fig. 1: A $(5,7)$-Dyck path
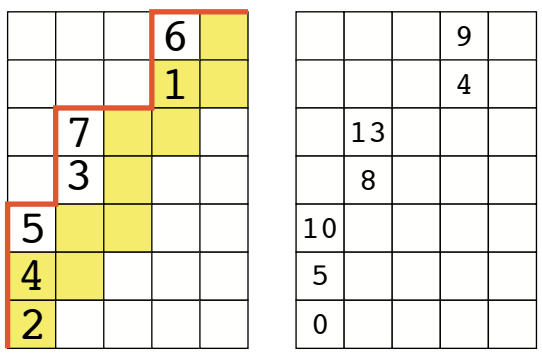

Fig. 2: A $(5,7)$-parking function and the ranks of its cars.

The number of full cells between an $(m, n)$-Dyck path $\Pi$ and the main diagonal is denoted area $(\Pi)$. For example, the Dyck path in Figure 1 has area 4. Notice that the collection of cells above a Dyck path $\Pi$ forms an english Ferrers diagram $\lambda(\Pi)$. For any cell $c \in \lambda(\Pi)$, let $\operatorname{leg}(c)$ and $\operatorname{arm}(c)$ denote the number of cells in $\lambda(\Pi)$ which are strictly south or strictly east of $c$, respectively. Then set

$$
\operatorname{dinv}(\Pi)=\sum_{c \in \lambda(\Pi)} \chi\left(\frac{\operatorname{arm}(c)}{\operatorname{leg}(c)+1}<\frac{m}{n}<\frac{\operatorname{arm}(c)+1}{\operatorname{leg}(c)}\right)
$$

The Dyck path in Figure 1 has $\lambda=(3,3,1,1)$. The 7 cells which contribute to dinv are marked.

As in the classical case, an $(m, n)$-parking Function PF is obtained by labeling the cells east of and adjacent to north steps of an $(m, n)$-Dyck path with $1,2, \ldots, n$ in a column-increasing way. This underlying Dyck path will be denoted $\Pi(\mathrm{PF})$. This is analogous to the visualization of classical parking functions which originated with Garsia and Haiman (1996). Classical parking functions correspond to preference functions which park cars on a one-way street (see Konheim and Weiss (1966)). We will borrow the term cars for the labels of the north steps. For brevity, let area $(\mathrm{PF})=\operatorname{area}(\Pi(\mathrm{PF}))$.

The definition of $\operatorname{dinv}(\mathrm{PF})$ and $\sigma(\mathrm{PF})$ will be more complex. In the classical case, these statistics are related to the idea of grouping cars by diagonals. Here, we extend this notion by using the function 


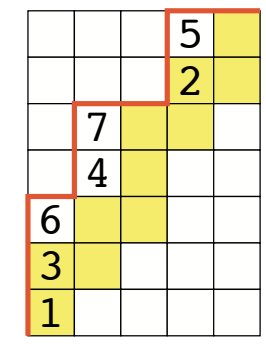

Fig. 3: A $(5,7)$-parking function with maximal tdinv.

$\operatorname{rank}(x, y)=m y-n x$. A point which is on the main diagonal has rank $=0$. In fact, rank orders points according to their distance from the main diagonal. We say that the rank of a car is the rank of the southwest corner of its cell. Figure 2 contains a $(5,7)$-parking function and the ranks of its cars.

In the classical case, the word of the parking function $\sigma$ is obtained by reading the cars from highest to lowest diagonal. Here, the word $\sigma$ of an $(m, n)$-parking function is obtained reading the cars from highest to lowest value of rank. In Figure 2, we have $\sigma=7563412$. As in the classical case, we will be interested in the descent set of the inverse of this permutation, ides(PF). In Figure 2, we have ides $=\{2,4,6\}$.

Furthermore, in the classical case, pairs of cars create diagonal inversions, or dinv, when their diagonals are not too far apart (and the cars occur in a certain order). Similarly, for an $(m, n)$-parking function PF, we set

$$
\operatorname{tdinv}(\mathrm{PF})=\sum_{\operatorname{cars} i<j} \chi(\operatorname{rank}(i)<\operatorname{rank}(j)<\operatorname{rank}(i)+m) .
$$

This is called the temporary dinv, or tdinv, because it needs to be modified to obtain dinv. In Figure 2 , the pairs of cars contributing to tdinv are $(1,3),(1,4),(3,5),(3,6),(4,6),(5,7)$, and $(6,7)$.

The final ingredient for dinv is

$$
\operatorname{maxtdinv}(\mathrm{PF})=\max \left\{\operatorname{tdinv}\left(\mathrm{PF}^{\prime}\right): \Pi\left(\mathrm{PF}^{\prime}\right)=\Pi(\mathrm{PF})\right\} .
$$

Now we can finally set

$$
\operatorname{dinv}(\mathrm{PF})=\operatorname{tdinv}(\mathrm{PF})+\operatorname{dinv}(\Pi(\mathrm{PF}))-\operatorname{maxtdinv}(\mathrm{PF}) .
$$

Note that maxtdinv $(\mathrm{PF})$ and $\operatorname{dinv}(\Pi(\mathrm{PF}))$ only depend on the underlying Dyck path.

It is fairly straight-forward to show that $\operatorname{maxtdinv}(\mathrm{PF})$ is simply tdinv of the parking function with underlying Dyck path $\Pi(\mathrm{PF})$ whose word is the reverse permutation $n \ldots 21$. The maximizing parking function corresponding to Figure 2 can be found in Figure 3 . Note that the tdinv of this parking function is 10. Combining this with our earlier observations, we have that the dinv of the parking function shown in Figure 2 is $7+7-10=4$.

Let $\mathcal{P} \mathcal{F}_{m, n}$ denote the set of all $(m, n)$-parking functions. For any subset $S \subset\{1,2, \ldots, n-1\}$, $F_{S}[X]$ denotes the corresponding fundamental quasi-symmetric function of Gessel (1984) which is ho- 
mogeneous of degree $n$. We now have the tools to define the Hikita polynomial of Hikita (2012):

$$
\mathrm{H}_{m, n}[X ; q, t]=\sum_{\mathrm{PF} \in \mathcal{P} \mathcal{F}_{m, n}} t^{\operatorname{area}(\mathrm{PF})} q^{\operatorname{dinv}(\mathrm{PF})} F_{\mathrm{ides}(\mathrm{PF})}[X] .
$$

Let $\nabla$ be the Macdonald polynomial eigen-operator of Bergeron and Garsia (1999). The Shuffle Conjecture of Haglund et al. (2005) can be restated as follows.

Conjecture 1 (Haglund-Haiman-Loehr-Remmel-Ulyanov) For all $n \geq 0$,

$$
\mathrm{H}_{n+1, n}(X ; q, t)=\nabla e_{n} .
$$

Gorsky and Negut (2013) extend this conjecture, giving a symmetric function expression for each coprime pair $(m, n)$ which conjecturally coincides with $\mathrm{H}_{m, n}(X ; q, t)$. The main actors in the symmetric function side of the Gorsky-Negut conjecture are the operators $D_{k}$ for each integer $k$, which were introduced in Garsia et al. (1999). Their action on a symmetric function $F[X]$ is defined by setting

$$
D_{k} F[X]=\left.F\left[X+\frac{M}{z}\right] \sum_{i \geq 0}(-z)^{i} e_{i}[X]\right|_{z^{k}}
$$

where $M=(1-t)(1-q)$.

We use here with a concrete realization of an algebra extensively studied by Schiffmann and Vasserot (2011). We will construct a family of symmetric function operators $\mathrm{Q}_{a, b}$ for any pair of integers $(a, b)$. For our purposes, we only need these operators when $a$ and $b$ are positive. It will be convenient to use the notation $\mathrm{Q}_{k m, k n}$ with $(m, n)$ coprime.

For any $n \geq 0$, set

$$
\mathrm{Q}_{1, n}=D_{n}
$$

Then we will recursively define $\mathrm{Q}_{m, n}$ as follows for $m>1$. Consider the $m \times n$ lattice with diagonal $y=$ $\frac{n}{m} x$. Let $(a, b)$ be the lattice point which is closest to and below the diagonal. Set $(c, d)=(m-a, n-b)$. We will write $\operatorname{Split}(m, n)=(a, b)+(c, d)$. Then let

$$
\mathrm{Q}_{m, n}=\frac{1}{M}\left[\mathrm{Q}_{c, d}, \mathrm{Q}_{a, b}\right]=\frac{1}{M}\left(\mathrm{Q}_{c, d} \mathrm{Q}_{a, b}-\mathrm{Q}_{a, b} \mathrm{Q}_{c, d}\right) .
$$

Note that any point of the form $(k x, k y)$ is further from the diagonal than the point $(x, y)$. Hence we can guarantee that the coordinates of $(a, b)$ are coprime whenever $\operatorname{Split}(m, n)=(a, b)+(c, d)$. The coordinates of $(c, d)$ are also coprime by a similar argument. Therefore all of the operators $\mathrm{Q}_{m, n}$ with $(m, n)$ coprime are defined by this recursion.

For example, $\operatorname{Split}(3,5)=(2,3)+(1,2)$ so that

$$
\mathrm{Q}_{3,5}=\frac{1}{M}\left[\mathrm{Q}_{1,2}, \mathrm{Q}_{2,3}\right] \text {. }
$$

The same procedure gives $\mathrm{Q}_{2,3}=\frac{1}{M}\left[\mathrm{Q}_{1,2}, \mathrm{Q}_{1,1}\right]$. Therefore

$$
\mathrm{Q}_{3,5}=\frac{1}{M^{2}}\left[D_{2},\left[D_{2}, D_{1}\right]\right]=\frac{1}{M^{2}}\left(D_{2} D_{2} D_{1}-2 D_{2} D_{1} D_{2}+D_{1} D_{2} D_{2}\right) .
$$

This geometric definition of $\operatorname{Split}(m, n)$ can be made algebraic. In particular, the set of vertical distances between the diagonal and the highest points $(x, y)$ below it, $1 \leq x \leq m$, must be contained in 
the set $\left\{\frac{i}{m}: 1 \leq i \leq m\right\}$. The coprimality of $(m, n)$ guarantees that these $m$ values are all distinct, so each value $\frac{i}{m}$ occurs exactly once. Therefore the closest point corresponds to the value $\frac{1}{m}$. Hence we can identify $(a, b)$ as the unique lattice point satisfying $n a-m b=1$ with $1 \leq a \leq m$.

The $(m, n)$-Shuffle Conjecture of Gorsky and Negut (2013) may be simply stated as follows.

Conjecture 2 (Gorsky-Negut) For all pairs of coprime positive integers $(m, n)$, we have

$$
\mathrm{Q}_{m, n}(-1)^{n}=\mathrm{H}_{m, n}[X ; q, t] .
$$

We will show in Section 2 that when $m=n+1$ this reduces to Conjecture 1

In the remainder of the paper we prove the following special cases of Conjecture 2

Theorem 1 For any $k \geq 0$,

$$
\mathrm{Q}_{2 k+1,2} 1=\mathrm{H}_{2 k+1,2}[X ; q, t] \quad \text { and } \quad \mathrm{Q}_{2,2 k+1}(-1)=\mathrm{H}_{2,2 k+1}[X ; q, t] \text {. }
$$

\section{The Symmetric Function Side}

To prove the symmetric function side of the first equation in Theorem 1 , and to understand why Conjecture 1 is a special case of Conjecture 2, we need the following lemma.

Lemma 1 For any positive, coprime $m, n$,

$$
\nabla \mathrm{Q}_{m, n} \nabla^{-1}=\mathrm{Q}_{m+n, n}
$$

The proof is by induction using the algebraic definition of Split. Hence we have that $\mathrm{Q}_{n+1, n}(-1)^{n}=$ $\nabla D_{n} \nabla^{-1}(-1)^{n}$. Since $\nabla^{-1}(-1)^{n}=(-1)^{n}$ and $D_{n}(-1)^{n}=e_{n}$, we see that Conjecture 1 is indeed a special case of Conjecture 2

Furthermore, our calculations for second equation in Theorem 1 make significant use of plethystic substitution. For the sake of completeness, we will review this idea here briefly. If $E=E\left(t_{1}, t_{2}, \ldots\right)$ is a formal Laurent series in the variables $t_{1}, t_{2}, \ldots$, we define

$$
p_{k}[E]=E\left(t_{1}^{k}, t_{2}^{k}, \ldots\right) \text {. }
$$

More generally, if $F$ is any symmetric function, it can be expressed as a polynomial $F=Q\left(p_{1}, p_{2}, \ldots\right)$ in the power basis. This given, we define

$$
F[E]=Q\left(p_{1}[E], p_{2}[E], \ldots\right) .
$$

Note that $p_{k}\left(x_{1}+x_{2}+\cdots+x_{n}\right)=p_{k}\left(x_{1}, x_{2}, \ldots, x_{n}\right)$. Hence for any symmetric function $F, F\left[x_{1}+\right.$ $\left.x_{2}+\cdots+x_{n}\right]=F\left(x_{1}, x_{2}, \ldots, x_{n}\right)$. We will write $X=x_{1}+x_{2}+\cdots+x_{n}$ whenever $n$ does not need to be specified so that $F[X]=F\left(x_{1}, x_{2}, \ldots, x_{n}\right)$ is the usual symmetric function.

\subsection{The case $n=2$}

The goal of this section is to calculate $\mathrm{Q}_{2 k+1,2} 1$ for any $k \geq 0$. Note that by Lemma $1 \mathrm{Q}_{2 k+1,2}=$ $\nabla^{k} \mathrm{Q}_{1,2} \nabla^{-k}$. Since this is applied to a constant, $\nabla^{-k}$ has no effect. Furthermore, $\mathrm{Q}_{1,2}=D_{2}$ and $D_{n} 1=(-1)^{n} e_{n}$ for any $n$. Therefore we have

$$
\mathrm{Q}_{2 k+1,2} 1=\nabla^{k} e_{2} \text {. }
$$


Calculating $\nabla^{k} e_{2}$ for a few small values of $k$ and expanding in terms of Schur functions will naturally lead to the conjecture

$$
\nabla^{k} e_{2}=\frac{\left(t^{k}-q^{k}\right)}{(t-q)} s_{2}+\frac{\left(t^{k+1}-q^{k+1}\right)}{(t-q)} s_{1,1}
$$

Note that the value $\frac{\left(t^{n}-q^{n}\right)}{(t-q)}$ is a $q, t$-analog of the number $n$. Here and after, we will write it as simply $[n]_{q, t}$ so that 16 becomes

$$
\nabla^{k} e_{2}=[k]_{q, t} s_{2}+[k+1]_{q, t} s_{1,1} .
$$

Then (17) can be proven by a simple induction. The base case is just one instance of the fact that $s_{1^{n}}=e_{n}$. For the inductive step, use the expansions

$$
s_{2}=\frac{t}{t-q} \widetilde{H}_{2}-\frac{q}{t-q} \widetilde{H}_{1,1} \quad \text { and } \quad s_{1,1}=\frac{1}{t-q} \widetilde{H}_{1,1}-\frac{1}{t-q} \widetilde{H}_{2}
$$

along with the fact that $\nabla$ is an eigenvector of $\widetilde{H}_{2}$ and $\widetilde{H}_{1,1}$ with eigenvalues $q$ and $t$ respectively. The remainder of the calculations are left to the reader.

\subsection{The case $m=2$}

Determining a simple formula for $\mathrm{Q}_{2,2 k+1}(-1)$ will require more familiarity with plethystic substitution. Note that $\operatorname{Split}(2,2 k+1)=(1, k)+(1, k+1)$. Hence $\mathrm{Q}_{2,2 k+1}=\frac{1}{M}\left[D_{k+1}, D_{k}\right]$. Therefore we will need to be able to calculate $D_{a} D_{b}(-1)$ for certain values of $a$ and $b$. Applying (7), we have

$$
\begin{aligned}
D_{a} D_{b}(-1) & =D_{a}\left((-1)^{b+1} e_{b}[X]\right) \\
& =\left.(-1)^{b+1} e_{b}\left[X+\frac{M}{z}\right] \sum_{j \geq 0}(-z)^{j} e_{j}[X]\right|_{z^{a}}
\end{aligned}
$$

To evaluate $e_{b}\left[X+\frac{M}{z}\right]$ we will use the additivity of the elementary symmetric functions. That is, for any $n$ and any expressions $E_{1}$ and $E_{2}$,

$$
e_{n}\left[E_{1}+E_{2}\right]=\sum_{k=0}^{n} e_{k}\left[E_{1}\right] e_{n-k}\left[E_{2}\right] .
$$

Furthermore, the elementary symmetric functions are homogeneous so

$$
e_{n}\left[\frac{M}{z}\right]=z^{-n} e_{n}[M]
$$

Using additivity, it is not too difficult to show that

$$
e_{i}[M]=\left\{\begin{array}{ll}
1 & \text { if } i=0 \\
(-1)^{i+1} M[i]_{q, t} & \text { if } i>0
\end{array} .\right.
$$


Combining these facts (19) becomes

$$
\begin{aligned}
D_{a} D_{b}(-1) & =\left.(-1)^{b+1} \sum_{i=0}^{b} e_{b-i}[X] e_{i}[M] z^{-i} \sum_{j \geq 0}(-z)^{j} e_{j}[X]\right|_{z^{a}} \\
& =\left.\sum_{i=0}^{b} \sum_{j \geq 0}(-1)^{j+b+1} e_{b-i}[X] e_{i}[M] e_{j}[X] z^{j-i}\right|_{z^{a}} \\
& =\sum_{u=0}^{b}(-1)^{a+b+i+1} e_{b-i}[X] e_{i}[M] e_{a+i}[X] \\
& =(-1)^{a+b+1} e_{b} e_{a}+(-1)^{a+b} M \sum_{i=1}^{b}[i]_{q, t} e_{b-i} e_{a+i}
\end{aligned}
$$

For our purposes, we will either have $a=k$ and $b=k+1$ or $a=k+1$ and $b=k$. Substituting into (23), we have

$$
D_{k} D_{k+1}(-1)=e_{k+1} e_{k}-M \sum_{i=1}^{k+1}[i]_{q, t} e_{k+1-i} e_{k+i}
$$

and

$$
D_{k+1} D_{k}(-1)=e_{k} e_{k+1}-M \sum_{i=1}^{k}[i]_{q, t} e_{k-i} e_{k+1+i} .
$$

Combining these gives

$$
\begin{aligned}
\mathrm{Q}_{2,2 k+1}(-1) & =\frac{1}{M}\left(D_{k+1} D_{k}(-1)-D_{k} D_{k+1}(-1)\right) \\
& =-\sum_{i=1}^{k}[i]_{q, t} e_{k-i} e_{k+1+i}+\sum_{i=1}^{k+1}[i]_{q, t} e_{k+1-i} e_{k+i} .
\end{aligned}
$$

Recall that $e_{n}=s_{1^{n}}$. The Littlewood-Richardson rule is a well-known procedure to calculate the product of two Schur functions, such as two elementary symmetric functions (see Macdonald (1995)). Using this rule, for any $k$ and any $i \geq 1$ we have

$$
e_{k+1-i} e_{k+i}=\sum_{j=0}^{k+1-i} s_{2^{k+1-i-j}, 1^{2 i+2 j-1}} \quad \text { and } \quad e_{k-i} e_{k+1+i}=\sum_{j=0}^{k-i} s_{2^{k-i-j}, 1^{2 i+2 j+1}} .
$$

Then 26 becomes

$$
\mathrm{Q}_{2,2 k+1}(-1)=-\sum_{i=1}^{k}[i]_{q, t} \sum_{j=0}^{k-i} s_{2^{k-i-j}, 1^{2 i+2 j+1}}+\sum_{i=1}^{k+1}[i]_{q, t} \sum_{j=0}^{k+1-i} s_{2^{k+1-i-j}, 1^{2 i+2 j-1}} .
$$

Note that

$$
\sum_{j=0}^{k-i} s_{2^{k-i-j}, 1^{2 i+2 j+1}}=\sum_{u=i}^{k} s_{2^{k-u}, 1^{2 u+1}}=\sum_{j=1}^{k+1-i} s_{2^{k+1-i-j}, 1^{2 i+2 j-1}}
$$




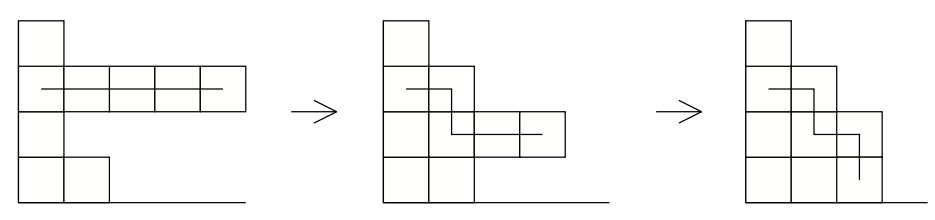

Fig. 4: The slinky rule gives $s_{(2,1,5,1)}=-s_{(2,4,2,1)}=s_{(3,3,2,1)}$

by the replacements $i+j \rightarrow u$ and $u \rightarrow i+j-1$. Hence we have

$$
\mathrm{Q}_{2,2 k+1}(-1)=[k+1]_{q, t} s_{1^{2 k+1}}+\sum_{i=1}^{k}[i]_{q, t} s_{2^{k+1-i}, 1^{2 i-1}}=\sum_{i=0}^{k}[k+1-i]_{q, t} s_{2^{i}, 1^{2 k-2 i+1}} .
$$

\section{The Combinatorial Side}

The goal of this section is to study the Hikita polynomials $\mathrm{H}_{2 k+1,2}(X ; q, t)$ and $\mathrm{H}_{2,2 k+1}(X ; q, t)$ for any $k \geq 0$. Hikita (2012) showed that $\mathrm{H}_{m, n}(X ; q, t)$ is symmetric (in $X$ ) for any coprime $m, n$. Therefore we should be able to expand these polynomials in terms of a symmetric function basis, rather than the Gessel quasisymmetric functions. Egge et al. (2010) give us a way to do precisely that. In particular,

Proposition 1 If $F=\sum_{S} c_{S} F_{S}$ is symmetric, then $F=\sum_{S} c_{S} s_{\rho(S)}$.

Here $\rho(S)$ is the composition of $n$ corresponding to $S \subset\{1,2, \ldots, n-1\}$, so that if $S=\left\{i_{1}<i_{2}<\right.$ $\left.\cdots<i_{k}\right\}$ then $\rho(S)=\left(i_{1}, i_{2}-i_{1}, \ldots, i_{k}-i_{k-1}, n-i_{k}\right)$.

The relationship between a Schur function indexed by a composition and the traditional partitionindexed Schur functions is given by the Murnaghan-Nakayama rule (see Macdonald (1995)). We give a brief overview of this rule here. It is also sometimes called the slinky rule for reasons that will be made clear below. For these purposes it is more useful to visualize partitions as french Ferrers diagrams.

Let $\rho=\left(\rho_{1}, \ldots, \rho_{k}\right)$ be a composition of $n$. Suppose that for some $i, \rho_{i}<\rho_{i+1}$ (i.e. $\rho$ is not already a partition). Then $s_{\rho}=-s_{\left(\rho_{1}, \ldots, \rho_{i+1}-1, \rho_{i}+1, \ldots, \rho_{k}\right)}$. Repeatedly applying this procedure will eventually yield a partition or a composition $\rho^{\prime}$ such that $\rho_{j}^{\prime}=\rho_{j+1}^{\prime}-1$ for some $j$. In the latter case, applying the slinky rule at $i=j$ yields $s_{\rho^{\prime}}=-s_{\rho^{\prime}}$, hence $s_{\rho^{\prime}}=0$. We can visualize this process as dropping the long segment of the composition (the slinky) over a staircase. Then the sign changes according to how many additional rows the slinky occupies after it falls. For example in Figure 4 , the slinky of length 5 ends up occupying an additional 2 rows in the resulting partition, giving a sign change of $(-1)^{2}$.

For brevity, we will write $\rho(\mathrm{PF})$ instead of $\rho(\operatorname{ides}(\mathrm{PF}))$ for a parking function $P F$. By Proposition 1 . we have

$$
\mathrm{H}_{m, n}(X ; q, t)=\sum_{\mathrm{PF} \in \mathcal{P F}_{m \times n}} t^{\operatorname{area}(\mathrm{PF})} q^{\operatorname{dinv}(\mathrm{PF})} s_{\rho(\mathrm{PF})} .
$$

With this simplification in mind, we will show that $\mathrm{H}_{2 k+1,2}(X ; q, t)$ and $\mathrm{H}_{2,2 k+1}(X ; q, t)$ agree with the formulas for $\mathrm{Q}_{2 k+1,2} 1$ and $\mathrm{Q}_{2,2 k+1}(-1)$ given in Section 2 


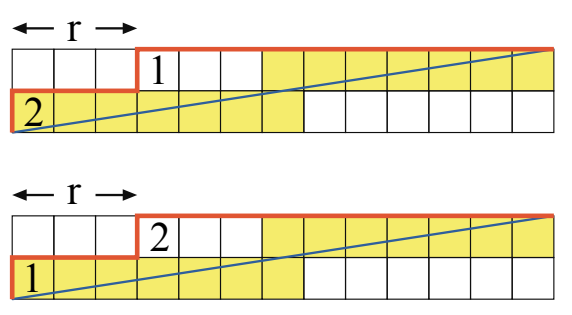

Fig. 5: Two fillings of a $(2 k+1,2)$-Dyck path.

\subsection{The case $n=2$}

There are $k+1$ possible Dyck paths in the $2 k+1 \times 2$ lattice. See Figure 5 . Each of these have two possible parking functions, except when $r=0$. Denote these by $\mathrm{PF}_{r}$ and $\mathrm{PF}_{r}$ respectively. For any $r$, we have that the rank of the car in the top row is $\operatorname{rank}(r, 1)=2 k+1-2 r$ and the rank of the car in the bottom row is 0 . Since $r \leq k$, the higher car always has the larger rank. Therefore for any $r, \sigma\left(\mathrm{PF}_{r}\right)=12$ and $\sigma\left(\mathrm{PF}_{r}^{\prime}\right)=21$. Correspondingly, $\rho\left(\mathrm{PF}_{r}\right)=(2)$ and $\rho\left(\mathrm{PF}_{r}^{\prime}\right)=(1,1)$. Both of these are already partitions, so we need not apply the slinky rule.

Furthermore, we clearly have area $\left(\mathrm{PF}_{r}\right)=\operatorname{area}\left(\mathrm{PF}_{r}^{\prime}\right)=k-r$. Each cell $c$ above their common Dyck path has $\operatorname{leg}(c)=0$ and $0 \leq \operatorname{arm}(c)<r \leq k$. Hence

$$
\frac{\operatorname{arm}(c)}{\operatorname{leg}(c)+1}<k<\frac{2 k+1}{2}<\infty=\frac{\operatorname{arm}(c)+1}{\operatorname{leg}(c)} .
$$

This gives $\operatorname{dinv}\left(\Pi\left(\mathrm{PF}_{r}\right)\right)=\operatorname{dinv}\left(\Pi\left(\mathrm{PF}_{r}^{\prime}\right)\right)=r$.

Now, in $\mathrm{PF}_{r}$ we have $2 k+1-2 r=\operatorname{rank}(1)>\operatorname{rank}(2)=0$ so $\operatorname{tdinv}\left(\mathrm{PF}_{r}\right)=0$. But in $\mathrm{PF}_{r}^{\prime}$, $\operatorname{rank}(1)=0$ and $\operatorname{rank}(2)=2 k+1-2 r$ so $\operatorname{rank}(1)<\operatorname{rank}(2)<\operatorname{rank}(1)+2 k+1$ unless $r=0$. Thus for $0<r \leq k, \operatorname{tdinv}\left(\mathrm{PF}_{r}\right)=0$ and $\operatorname{tdinv}\left(\mathrm{PF}_{r}^{\prime}\right)=1$, while $\operatorname{tdinv}\left(\mathrm{PF}_{0}^{\prime}\right)=0$. Thus maxtdinv $\left(\mathrm{PF}_{r}\right)=$ $\operatorname{maxt} \operatorname{dinv}\left(\mathrm{PF}_{r}^{\prime}\right)=1$ for $0<r \leq k$ and $\operatorname{maxtdinv}\left(\mathrm{PF}_{0}^{\prime}\right)=0$.

Combining this information gives $\operatorname{dinv}\left(\mathrm{PF}_{r}\right)=r-1$ and $\operatorname{dinv}\left(\mathrm{PF}_{r}^{\prime}\right)=r$ for all $r$. Therefore we have

$$
\begin{aligned}
\mathrm{H}_{2 k+1,2}(X ; q, t) & =\sum_{r=1}^{k} t^{k-r} q^{r-1} s_{(2)}+\sum_{r=0}^{k} t^{k-r} q^{r} s_{(1,1)} \\
& =[k]_{q, t} s_{(2)}+[k+1]_{q, t} s_{(1,1)} .
\end{aligned}
$$

This result, in conjunction with (17), completes the proof of the first equation in Theorem 1 .

\subsection{The case $m=2$}

Unlike the case $n=2$, the case $m=2$ addresses a large number of parking functions. In order to reduce our computations, then, we need the lemma below. The following corollary will then reveal that the majority of the parking functions will contribute nothing to $\mathrm{H}_{2, n}(X ; q, t)$.

Lemma 2 For any rational parking function $\mathrm{PF}$ with cars in $k$ columns, $\rho(\mathrm{PF})$ consists only of the numbers $\{1,2, \ldots, k\}$. 
Proof: Recall that $\sigma(\mathrm{PF})(i)=j$ means that position $i$ contains car $j$. On the other hand, $\sigma(\mathrm{PF})^{-1}(j)=i$ means that car $j$ is in position $i$. If $\rho(\mathrm{PF})$ contains a part of size $r$, then there is some $j$ so that

$$
\sigma(\mathrm{PF})^{-1}(j)<\sigma(\mathrm{PF})^{-1}(j+1)<\cdots<\sigma(\mathrm{PF})^{-1}(j+r-1) .
$$

However if any two of these cars are in the same column, they create a descent (since the smaller car is lower and therefore has lower rank). Hence there must be at least $r$ columns containing cars of PF.

Corollary 1 For any $k \geq 0$ and $\mathrm{PF} \in \mathcal{P F}_{2 \times 2 k+1}$, either $s_{\rho(\mathrm{PF})}$ is 0 or there is some $r \geq 0$ such that $\rho(\mathrm{PF})=\left(2^{r}, 1^{2 k+1-2 r}\right)$ and $\operatorname{ides}(\mathrm{PF})=\{2,4, \ldots, 2 r, 2 r+1, \ldots, 2 k\}$.

Proof: By the previous lemma, $\rho(\mathrm{PF})$ consists of only 1's and 2's. Applying the slinky rule to $s_{\rho(\mathrm{PF})}$ then gives 0 unless $\rho(\mathrm{PF})$ is already the partition $\left(2^{r}, 1^{2 k+1-2 r}\right)$ for some $r \geq 0$. In this case, $\rho(\mathrm{PF})$ can only correspond to the desired set ides $(\mathrm{PF})$.

Hence finding the coefficient of $s_{\left(2^{r}, 1^{2 k+1-2 r}\right)}$ in $\mathrm{H}_{2,2 k+1}(X ; q, t)$ is equivalent to finding all parking functions with ides $=\{2,4, \ldots, 2 r, 2 r+1, \ldots, 2 k\}$. The proof of Lemma 2 shows that for all $1 \leq l \leq r$ such a parking function must have the cars $2 l-1$ and $2 l$ in different columns. Suppose that a Dyck path $\Pi$ has at least $r$ north steps in each column. Then there is exactly one parking function supported by $\Pi$ with the desired inverse descent set. In particular, 1 and 2 must be the lowest cars in each column, in whichever columns give $\sigma^{-1}(1)<\sigma^{-1}(2)$. Similarly, for each $1 \leq l \leq r, 2 l-1$ and $2 l$ are the $l$ th lowest cars in each column, and which car goes in which column is determined. Then the remaining cars must be placed in decreasing order so that $\sigma^{-1}$ has descents at $2 r, 2 r+1, \ldots, 2 k$.

Since there is only one Dyck path with a given area, we have just one parking function which has $\rho=\left(2^{r}, 1^{2 k+1-2 r}\right)$ and area $=k-r-u$ for any given $0 \leq r \leq k$ and $0 \leq u \leq k-r$. That is, the Dyck path has exactly $r+u$ north steps in the second column (giving area $=k-r-u$ ) and is decorated by cars as indicated above to yield a parking function with the specified composition $\rho$. Denote this Dyck path by $\Pi^{(r, u)}$ and the parking function by $\mathrm{PF}^{(r, u)}$. Therefore we have

$$
\begin{aligned}
\mathrm{H}_{2,2 k+1}(X ; q, t) & =\sum_{r=0}^{k} \sum_{u=0}^{k-r} t^{\operatorname{area}\left(\mathrm{PF}^{(r, u)}\right)} q^{\operatorname{dinv}\left(\mathrm{PF}^{(r, u)}\right)} s_{\rho\left(\mathrm{PF}^{(r, u)}\right)} \\
& =\sum_{r=0}^{k} \sum_{u=0}^{k-r} t^{k-r-u} q^{\operatorname{dinv}\left(\mathrm{PF}^{(r, u)}\right)} s_{\left(2^{r}, 1^{2 k+1-2 r}\right)}
\end{aligned}
$$

On the other hand, 30 gives

$$
\begin{aligned}
\mathrm{Q}_{2,2 k+1}(-1) & =\sum_{r=0}^{k}[k+1-r]_{q, t} s_{\left(2^{r}, 1^{2 k+1-2 r}\right)} \\
& =\sum_{r=0}^{k} \sum_{u=0}^{k-r} t^{k-r-u} q^{u} s_{\left(2^{r}, 1^{2 k+1-2 r}\right)}
\end{aligned}
$$

Hence if we can show that $\operatorname{dinv}\left(\mathrm{PF}^{(r, u)}\right)=u$, we will have proven the second equation in Theorem 1 . 


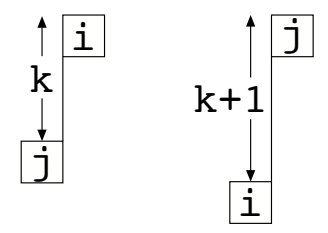

Fig. 6: Possible placements of cars $i, j$ such that $\operatorname{rank}(j)=\operatorname{rank}(i)+1$.

Note that $\operatorname{dinv}\left(\Pi\left(\mathrm{PF}^{(r, u)}\right)\right)=r+u$. This is because each cell $c$ above $\Pi^{(r, u)}$ has $\operatorname{arm}(c)=0$ and $0 \leq \operatorname{leg}(c)<r+u \leq k$ and therefore

$$
0=\frac{\operatorname{arm}(c)}{\operatorname{leg}(c)+1}<\frac{2}{2 k+1}<\frac{1}{k}<\frac{\operatorname{arm}(c)+1}{\operatorname{leg}(c)} .
$$

We can also see that maxtdinv $\left(\mathrm{PF}^{(r, u)}\right)=2 r+2 u$. This is because tdinv counts pairs of cars $i<j$ such that

$$
\operatorname{rank}(i)<\operatorname{rank}(j)<\operatorname{rank}(i)+2 \Rightarrow \operatorname{rank}(j)=\operatorname{rank}(i)+1 .
$$

All of the ranks of a given column have the same parity. In fact, the first column must have cars of rank $0,2, \ldots, 2 k$, while the second column can only have cars of rank $1,3, \ldots, 2 k-1$. Hence each of the $r+u$ cars $a$ of the second column have two cars $b$ in the first column with $\operatorname{rank}(b)=\operatorname{rank}(a) \pm 1$.

Note that moving down a row corresponds to subtracting 2 from the rank, while moving right subtracts $2 k+1$. The relative positions of cars $i<j$ contributing to tdinv are shown in Figure 6 We will such pairs in $\mathrm{PF}^{(r, u)}$ according to cases.

Recall that the cars $2 r, 2 r+1, \ldots 2 k+1$ occupy all but the lowest $r$ positions in each column and exactly $u$ of them are in the second column. Suppose that $i$ is one of these $u$ cars. Then $i$ is in the $u$ th row of $\mathrm{PF}^{(r, u)}$ or higher. Moving down $k$ rows in the first column to find the car $j$ with $\operatorname{rank}(j)=\operatorname{rank}(i)+1$ (as seen in Figure 6) will still put $j$ more than $r$ rows from the bottom since $k+u+r<2 k+1$. Hence $j \geq 2 r$ as well. Furthermore, the cars $2 r, 2 r+1, \ldots 2 k+1$ are in increasing order according to rank. Therefore $i<j$. Hence each of these $s$ possible pairs $(i, j)$ contribute to tdinv.

If $j \geq 2 r$ is in the second column, each car larger than $j$ has a larger rank than $j$. Hence the corresponding $i$ (according to Figure 6) must be less than $j$, so these $u$ pairs also contribute to tdinv.

We also need to assess the contribution to tdinv of the other $r$ cars in the second column. Let $i$ be the $l$ th lowest car of the second column for some $1 \leq l \leq r$. The corresponding $j$ (according to Figure 6) is $k$ rows lower than $i$ in the first column. Thus it is in the $(2 k+1-r-u+l)-k$ th row from the bottom, which is higher than row $l$. Hence $j>i$, so each of the $r$ such pairs contributes to tdinv.

On the other hand, let $j$ be the $l$ th lowest car of the second column. Then the corresponding $i$ is $k+1$ rows lower than $j$ in the first column. Except in the case $r+u=k, i$ is higher in the first column than row $l$. Hence we would have to have $i>j$ and this pair would not contribute to tdinv. Now suppose that $r+u=k$. Then $i$ is in row $l$ of the first column. Thus $i$ and $j$ are the cars $2 l$ and $2 l-1$ in some order. These cars were placed in $\mathrm{PF}^{(r, u)}$ so that $\sigma^{-1}(2 l-1)<\sigma^{-1}(2 l)$. In other words, so that $\operatorname{rank}(2 l-1)>\operatorname{rank}(2 l)$. Thus it must be that case that $i=2 l$ and $j=2 l-1$ so that $i>j$. This pair also does not contribute to tdinv. 
Combining all of these cases gives $\operatorname{tdinv}\left(\mathrm{PF}^{(r, u)}\right)=r+2 u$. Therefore for each $r, u$,

$$
\operatorname{dinv}\left(\mathrm{PF}^{(r, u)}\right)=(r+u)+(r+2 u)-(2 r+2 u)=u .
$$

As observed above, this completes the proof that

$$
\begin{aligned}
\mathrm{H}_{2,2 k+1}(X ; q, t) & =\sum_{r=0}^{k} \sum_{u=0}^{k-r} t^{k-r-u} q^{u} s_{\left(2^{r}, 1^{2 k+1-2 r}\right)} \\
& =\mathrm{Q}_{2,2 k+1}(-1) .
\end{aligned}
$$

\section{References}

F. Bergeron and A. M. Garsia. Science Fiction and Macdonald's Polynomials. CRM Proceedings \& Lecture Notes, American Mathematical Society, 22:1-52, 1999.

E. Egge, N. Loehr, and G. Warrington. From quasisymmetric expansions to Schur expansions via a modified inverse Kostka matrix. European J. Comin., 31(8):390-394, 2010.

A. M. Garsia and M. Haiman. Some Natural Bigraded $S_{n}$-Modules and $q, t$-Kostka Coefficients. Electron. J. Combin., 3(2), 1996.

A. M. Garsia, M. Haiman, and G. Tesler. Explicit plethystic formulas for Macdonald $(q, t)$-Kostka coefficients. Séminaire Lotharingien de Combinatoire [electronic only], 42:B42m, 1999.

I. Gessel. Multipartite P-partitions and inner products of skew Schur functions. Contemp. Math, 34: 289-301, 1984.

E. Gorsky and A. Negut. Refined knot invariants and Hilbert schemes. arXiv preprint arXiv:1304.3328, 2013

J. Haglund, M. Haiman, N. Loehr, J. B. Remmel, and A. Ulyanov. A combinatorial formula for the character of the diagonal coinvariants. Duke J. Math., 126:195-232, 2005.

T. Hikita. Affine springer fibers of type a and combinatorics of diagonal coinvariants. arXiv preprint arXiv:1203.5878, 2012.

A. G. Konheim and B. Weiss. An occupancy discipline and applications. SIAM J. Appl. Math., 14(6): 1266-1274, 1966.

I. G. Macdonald. Symmetric functions and Hall polynomials. Oxford Mathematical Monographs, New York, 2 edition, 1995.

A. Negut. Moduli of Flags of Sheaves on $\mathbb{P}^{2}$ and their K-theory. arXiv preprint arXiv:1209.4242, 2012a.

A. Negut. The shuffle algebra revisited. arXiv preprint arXiv:1209.3349, $2012 \mathrm{~b}$.

O. Schiffmann and E. Vasserot. The elliptical Hall algebra, Cherednik Hecke algebras and Macdonald polynomials. Compos. Math., 147.1:188-234, 2011.

O. Schiffmann and E. Vasserot. The elliptical Hall algebra and the equivariant K-theory of the Hilbert scheme of $\mathbb{A}^{2}$. Duke J. Math., 162.2:279-366, 2013. 